\title{
Survival efficacy and potential of Mesorhizobium Ciceri for enhancing the growth and yield of imidacloprid-treated chickpea (Cicer arietinum)
}

\author{
Sajid Mahmood Nadeem*1, Muhammad Yahya Khan ${ }^{1}$, Maqshoof Ahmad ${ }^{2}$, Muhammad Naeem Mushtaq ${ }^{1}$, Kashif Nazir \\ Qureshi ${ }^{1}$ and Muhammad Naveed ${ }^{3}$ \\ ${ }^{1}$ University of Agriculture, Faisalabad, Sub-campus Burewala-Vehari, 61100, Pakistan \\ ${ }^{2}$ Department of Soil Science, University College of Agriculture and Environmental Sciences, the Islamia University of \\ Bahawalpur, Bahawalpur, 63100, Pakistan \\ ${ }^{3}$ Institute of Soil \& Environmental Sciences, University of Agriculture, Faisalabad, 38040, Pakistan
}

[Received: April 29, 2019 Accepted: June 28, 2019 Published Online: January 07, 2020]

\begin{abstract}
Chickpea relies on inoculation with $N_{2}$-fixing bacteria to fulfill its $N$ requirement. The efficacy of the inoculation process hangs on the success of association between $N_{2}$-fixing bacteria and Chickpea plants. Seed treatment with insecticides may kill the inoculated bacteria and result in poor establishment and upshot of the symbiosis. Survival efficacy of four rhizobial (Mesorhizobium ciceri) strains (CRI $I_{14}, C_{20} I_{20} C_{34}$ and $C R I_{35}$ ) selected on the basis of preliminary study, was evaluated at increasing concentration of imidacloprid $\left(0-200 \mu g L^{-1}\right)$. Amongst four inoculated strains, the strain CRI20 and CRI35 showed better survival in the presence of Imidacloprid when compared to rest of two. Strain $C R I_{35}$ was able to tolerate insecticide above the recommended dose whereas the strain $\mathrm{CRI}_{20}$ showed relatively less growth at high concentration. So, for as the efficacy of these strains was concerned pertaining to improvement in growth of imidacloprid treated chickpea it was tested in a pot trial. Chickpea seeds were treated with recommended dose of imidacloprid and then inoculated with respective rhizobial strains as per experimental plan. Un-inoculated treated and untreated seeds were also used for comparison. The pots were arranged according to completely randomized design in two sets having three replications each. At flowering, plants from one set were uprooted and data regarding nodulation was recorded, whereas, the data regarding growth and yield parameters was calculated from other set at maturity. The results showed that inoculation not only improved nodulation but also caused a significant increase in growth and yield. Rhizobial strain $C^{2} I_{35}$ performed better than the other which might be due to its better growth promoting traits in addition to its ability to tolerate insecticide. Therefore, it is suggested to use only those rhizoidal strains that could tolerate agrochemicals and survive well in the rhizosphere and would consequently nodulate legume plants more efficiently.
\end{abstract}

Keywords: Insecticide, legume, nodulation, tolerance, yield

\section{Introduction}

The bacteria referred to as rhizobia are able to form nodules on legume plants inside of which they fix nitrogen. Use of rhizobial strains is a widespread practice for enhancing soil fertility and improving crops yield (Li et al., 2013; Binyamin et al., 2019). In addition to symbiotic nitrogen fixation, Rhizobia also produce phytohormones that play important role in plant growth and development (Dubey et al., 2010; Sahasrabudhe, 2011). However, rhizobia are very sensitive to environmental change. The agrochemicals such as insecticides, fungicides and herbicides, are now used extensively in agriculture for enhancing crop production but they can also damage the environment and the activities of inoculant bacteria very adversely (Ahemad and Khan, 2010). Moreover, these toxic chemicals reduce the process of nitrogen fixation by affecting the signaling between legume derived phytochemicals and rhizobium Nod D receptors (Fox et al., 2007; Ahemad and Khan, 2011) or reducing the allocation of photosynthates to the nodules for $\mathrm{N}_{2}$ fixation (Koopman et al., 1995; Datta et al., 2009; Parween et al., 2016). Also, the recurrent use of agrochemicals results in accumulation/persistence of these chemicals to a level that is detrimental to the rhizobium survival and ultimately Rhizobium-legume association (Eliason et al., 2004; Wani et al., 2005; Ahemad and Khan, 2012a, 2013). The pesticides and their degraded products adversely affect the process of nitrogen fixation by causing negative impact on rhizobium growth therefore reducing the performance of agroecosystem (Pham et al., 2004; Anderson et al., 2004; Gaind et al., 2007).

\footnotetext{
*Email: smnadeem@uaf.edu.pk
} 
Among various legumes, chickpea (Cicer arietinum L.) is one of the most widely grown legumes in arid and semiarid regions. It is not only a good source of protein and vitamins (Ali et al., 2010) but also considered a suitable crop for improving soil nutrient status (Mohammadi et al., 2010). Rhizobium-legume symbiosis is one of the important associations for increasing nitrogen content of soil in semiarid regions of the world. To ensure effective nodulation and subsequent nitrogen fixation, the legumes are inoculated with suitable rhizobial strains (Zahir et al., 2018), however, without exhibiting tolerance against agrochemicals, the rhizobium could not be able to show its full potential for enhancing growth of inoculated plant. Therefore, to mitigate the negative impact of pesticides, it is necessary to inoculate the seeds with rhizobium which displays a wide range of tolerance against pesticides and exhibits its growth promoting traits for enhancing plant growth under pesticide stress (Wani et al., 2005; Ayansina, 2009; Ahemad and Khan, 2010).

The present study was therefore, designed to evaluate the survival of four rhizobial strains $\left(\mathrm{CRI}_{14}, \mathrm{CRI}_{20}, \mathrm{CRI}_{34}\right.$ and $\mathrm{CRI}_{35}$ ) against imidacloprid under axenic conditions. All of the four rhizobia were belonged to four different strains of Mesorhizobium ciceri. The efficacy of M. ciceri strains for enhancing growth and yield of insecticide (imidacloprid) treated chickpea was further evaluated by conducting a pot trial in green house.

\section{Materials and Methods}

A number of rhizobial strains were isolated from the root nodules of chickpea (Cicer arietinum) plants grown in pesticide-contaminated fields using yeast extract mannitol media (YEM). For this purpose, chickpea root samples were collected from experimental fields of the University of Agriculture, Faisalabad according to standard procedure. The nodules were separated from these samples and were surface sterilized by dipping in $95 \%$ ethanol solution momentarily followed by dipping in $0.2 \% \mathrm{HgCl}_{2}$ solution and then washings with sterilized water (Russel et al., 1982).

The surface sterilized nodules were crushed in a minimal volume of sterilized water and a loopful of the suspension was streaked out on yeast extract mannitol (YEM) agar medium (Vincent, 1970). Plates were incubated at $28 \pm 1{ }^{\circ} \mathrm{C}$ and well isolated, single colonies, shiny in appearance, were picked and re-streaked on fresh plates to obtain the pure cultures.

\section{Growth pouch trial}

To evaluate the potential of rhizobial isolates for enhancing growth of chickpea, an axenic study was conducted under controlled conditions. Broth of each isolate was prepared, and surface sterilized seeds (pesticide free seeds) of chickpea were inoculated by dipping in respective broth. Three inoculated seeds were placed in each sterilized (autoclaved) growth pouch. In case of control, seeds were dipped in sterilized broth. Growth pouches were placed in growth chamber and were arranged using a completely randomized design with three replications of each treatment. The light intensity in growth chamber was adjustedto350 $\mu \mathrm{mol} \mathrm{m} \mathrm{m}^{-2} \mathrm{~s}^{-1}$ with $8 \mathrm{~h}$ dark and $16 \mathrm{~h}$ light period and temperature was maintained at $25 \pm 2{ }^{\circ} \mathrm{C}$. For nutrition, sterilized Hoagland solution (half strength) was applied. After twenty-two (22) days, plants were harvested and data regarding shoot/root length and shoot/root fresh weight were collected. The data were analyzed statistically (Steel et al., 2007) and four strains $\left(\mathrm{CRI}_{14}, \mathrm{CRI}_{20}, \mathrm{CRI}_{34}\right.$ and $\left.\mathrm{CRI}_{35}\right)$ were selected for further study by General Linear Model (Mead et $a l ., 2003)$ using Minitab ${ }^{\mathrm{TM}}$ version 16 software. Rhizobial strains were confirmed as Mesorhizobium ciceri through host specificity test (Somasegaran and Hoben,1994).

\section{Insecticide tolerance of selected strains}

Four rhizobial strains $\left(\mathrm{CRI}_{14}, \mathrm{CRI}_{20}, \mathrm{CRI}_{34}\right.$ and $\left.\mathrm{CRI}_{35}\right)$ were tested for their ability to tolerate various concentration of imidacloprid $\left(0,50,100,150\right.$ and $\left.200 \mu \mathrm{g} \mathrm{L}^{-1}\right)$. For this purpose, broth of Yeast Extract Mannitol (YEM) media were prepared in flasks and amended separately with increasing concentration of imidacloprid $(0,50,100,150$ and $200 \mu \mathrm{g} \mathrm{L}^{-1}$ ). Then, these flasks were inoculated with each rhizobium strain repeated three times. The flasks were placed in shaking incubator at $28 \pm 2^{\circ} \mathrm{C}$. After 72 hours, the optical density was observed at $540 \mathrm{~nm}$. The strains which showed high optical density compared to other strains at increasing concentration of imidacloprid were considered as insecticide tolerant.

\section{Evaluating inoculation impact on insecticide treated chickpea in pot culture}

The effect of inoculation of rhizobia on chickpea treated with recommended dose of imidacloprid was evaluated by conducting a pot trial in green house. For this purpose, surface sterilized seeds of chickpea were treated with recommended dose (100 $\left.\mu \mathrm{g} \mathrm{L}^{-1}\right)$ of imidacloprid (Ahemad and Khan, 2012). The treated and untreated seeds were inoculated separately with freshly prepared culture of two rhizobial strains $\left(\mathrm{CRI}_{20}\right.$, and $\left.\mathrm{CRI}_{35}\right)$ that were already selected on the basis of their ability to tolerate high concentration of imidacloprid. Three seeds were sown in each pot of having twenty (20) kilogram sieved soil mixed with recommended dose of NPK fertilizer. Un-inoculated treated and untreated seeds were also sown for comparison. 
The pots were arranged according to completely randomized design. There were two sets of pots each having three replications. After emergence, uniform population of one plant was maintained in each pot. The water requirement of the plant was fulfilled with good quality water fulfilling the criteria of Ayers and Westcot (1985). At flowering, plants from one set were uprooted and data regarding number of nodules, nodule fresh weight and nodule dry weight were recorded. The data regarding growth and yield parameter were recorded from the second set at maturity. Root and shoot samples were analyzed for nitrogen and phosphorus content (Ryan et al. 2001). The collected data were subjected to statistical analysis according to Steel et al. (2007) using SPSS software version 19.

\section{Bacterial population in chickpea rhizosphere}

Population of bacteria (culturable counts) in chickpea rhizosphere was determined by agar plate dilution method. For this purpose, the rhizosphere soil samples from each treatment (treated with imidacloprid and untreated) were collected. One-gram rhizosphere soil was mixed with $9 \mathrm{~mL}$ of sterilized water. Then $1 \mathrm{~mL}$ of solution from this test tube was taken and added to another test tube with $9 \mathrm{~mL}$ sterilized water. This gives a dilution of $10^{-2}$ and in the same pattern dilutions up to $10^{-7}$ were prepared. From these dilutions, an aliquot of $100 \mu \mathrm{L}$ was spread plate method on nutrient plates and incubated for 48 hours in an incubated at $28^{\circ} \mathrm{C} \pm 2^{\circ} \mathrm{C}$. After incubation, bacterial colonies grown on nutrient agar medium were counted and bacterial populations were enumerated and expressed as colony farming unit per gram of soil (cfu g ${ }^{-1}$ of soil).

\section{Characterization of strains}

The selected rhizobial strains were characterized for their ability to colonize chickpea root, indole acetic acid production, phosphate solubilization, siderophores synthesis and exopolysaccharides production. Root colonization assay was determined by modified method of Simons et al. (1996). Phosphate solubilization ability of the rhizobial strains was observed on Pikovskaya's agar medium (Pikovskaya, 1948) containing tricalcium phosphate as insoluble phosphate source. The strains were examined for their ability to produce indole acetic acid using the method proposed by Brick et al. (1991). The method of Alexander and Zuberer (1991) was used to determine the ability of strain to synthesize siderophores qualitatively. Exopolysaccharides production was determined qualitatively by grown in growth medium at $150 \mathrm{rpm}$ at $25^{\circ} \mathrm{C}$. Supernatant was collated and then cold absolute ethylene was added drop wise under stirring and the formation of a precipitate was considered as positive for the production of exopolysaccharides (Nicolaus et al. 1999).

\section{Results}

\section{Axenic Trial}

Data regarding shoot/root length of chickpea showed that inoculation with rhizobial strains caused significant effect on these growth parameters (Table 1). Maximum shoot length (44\% more than un-inoculated control) was obtained with $\mathrm{CRI}_{20}$ followed by $\mathrm{CRI}_{34}$ and $\mathrm{CRI}_{35}$. Isolate $\mathrm{CRI}_{20}$ and $\mathrm{CRI}_{34}$ also caused maximum increase in root length that was $54 \%$ more than un-inoculated control and both rhizobial strains were statistically similar. Data regarding shoot weight also showed that inoculation with rhizobial isolates improved the weight compared to uninoculated control (Table 1). The most effective strain was $\mathrm{CRI}_{14}$ (46\% more shoot weight than control) followed by $\mathrm{CRI}_{34}$ and $\mathrm{CRI}_{20}$. Isolate $\mathrm{CRI}_{20}$ also caused maximum increase in root weight. Next effective strains were $\mathrm{CRI}_{34}$ and $\mathrm{CRI}_{35}$. On overall basis isolates $\mathrm{CRI}_{14}, \mathrm{CRI}_{20}, \mathrm{CRI}_{34}$ and $\mathrm{CRI}_{35}$ showed promising results during growth pouch study and selected for further study.

\section{Assessment of pesticide tolerance of rhizobial strains}

Four rhizobial strains were assessed for their potential to tolerate insecticide against five levels of imidacloprid. In the absence of imidacloprid, all strains showed good growth (Figure 1). At lower levels of pesticide $\left(0\right.$ and $\left.50 \mu \mathrm{g} \mathrm{L}^{-1}\right)$, the growth of all rhizobial strains remained unaffected. However, at recommended dose of imidacloprid $\left(100 \mu \mathrm{g} \mathrm{L}^{-}\right.$ ${ }^{1}$ ), statically significant reduction in growth of $\mathrm{CRI}_{14}$ and $\mathrm{CRI}_{34}$ strains was observed while the growth $\mathrm{CRI}_{20}$ and $\mathrm{CRI}_{35}$ strains remained almost at par of their growth at $0 \mu \mathrm{g}$ $\mathrm{L}^{-1}$ level of imidacloprid. However, the growth of $\mathrm{CRI}_{20}$ significantly decreased at high level $\left(150 \mu \mathrm{g} \mathrm{L}^{-1}\right)$ as compared to its growth at $0 \mu \mathrm{g} \mathrm{L}^{-1}$ level of imidacloprid. Further, the growth of $\mathrm{CRI}_{35}$ strain was also decreased at higher level, however, it was remained able to maintain its growth at highest concentration i.e. $200 \mu \mathrm{g} \mathrm{L}^{-1}$ of imidacloprid that showed its ability to tolerate high concentration of insecticide.

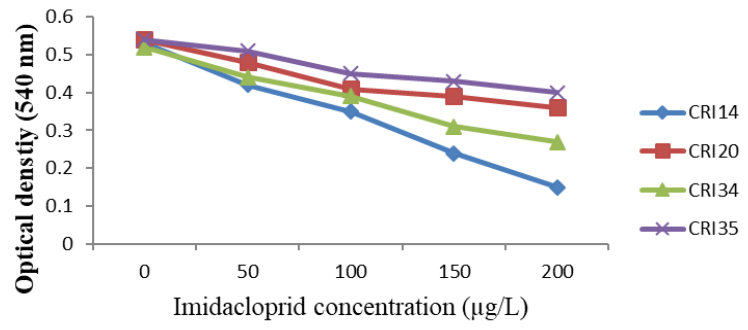

Figure 1: Effect of increasing rate of imidacloprid on growth of rhizobium strains 
Table 1. Effect of different rhizobial isolates on shoot/root length of chickpea

\begin{tabular}{|c|c|c|c|c|}
\hline Isolate & Shoot length (g/plant) & Root length (cm/plant) & Shoot weight (g/plant) & Root weight (g/plant) \\
\hline $\mathrm{CRI}_{1}$ & $16 \pm 0.14$ & $13 \pm 0.13$ & $0.48 \pm 0.05$ & $0.20 \pm 0.02$ \\
\hline $\mathrm{CRI}_{2}$ & $18 \pm 0.12$ & $15 \pm 0.14$ & $0.51 \pm 0.08$ & $0.23 \pm 0.03$ \\
\hline $\mathrm{CRI}_{3}$ & $16 \pm 0.10$ & $14 \pm 0.18$ & $0.55 \pm 0.09$ & $0.21 \pm 0.04$ \\
\hline $\mathrm{CRI}_{4}$ & $17 \pm 0.12$ & $15 \pm 0.11$ & $0.47 \pm 0.02$ & $0.24 \pm 0.02$ \\
\hline $\mathrm{CRI}_{5}$ & $18 \pm 0.21$ & $17 \pm 0.12$ & $0.49 \pm 0.03$ & $0.28 \pm 0.02$ \\
\hline $\mathrm{CRI}_{6}$ & $17 \pm 0.21$ & $14 \pm 0.21$ & $0.47 \pm 0.05$ & $0.20 \pm 0.03$ \\
\hline $\mathrm{CRI}_{7}$ & $19 \pm 0.22$ & $16 \pm 0.21$ & $0.49 \pm 0.06$ & $0.23 \pm 0.04$ \\
\hline $\mathrm{CRI}_{8}$ & $19 \pm 0.23$ & $17 \pm 0.23$ & $0.51 \pm 0.07$ & $0.27 \pm 0.01$ \\
\hline $\mathrm{CRI}_{9}$ & $15 \pm 0.21$ & $13 \pm 0.20$ & $0.60 \pm 0.07$ & $0.20 \pm 0.02$ \\
\hline $\mathrm{CRI}_{10}$ & $16 \pm 0.10$ & $14 \pm 0.19$ & $0.51 \pm 0.06$ & $0.19 \pm 0.02$ \\
\hline $\mathrm{CRI}_{11}$ & $18 \pm 0.20$ & $16 \pm 0.20$ & $0.52 \pm 0.08$ & $0.21 \pm 0.02$ \\
\hline $\mathrm{CRI}_{12}$ & $17 \pm 0.19$ & $15 \pm 0.18$ & $0.53 \pm 0.07$ & $0.23 \pm 0.01$ \\
\hline $\mathrm{CRI}_{13}$ & $16 \pm 0.13$ & $14 \pm 0.20$ & $0.54 \pm 0.09$ & $0.21 \pm 0.03$ \\
\hline${ }^{*} \mathrm{CRI}_{14}$ & $20 \pm 0.22$ & $18 \pm 0.12$ & $0.70 \pm 0.03$ & $0.32 \pm 0.03$ \\
\hline $\mathrm{CRI}_{15}$ & $21 \pm 0.11$ & $18 \pm 0.14$ & $0.56 \pm 0.11$ & $0.31 \pm 0.01$ \\
\hline $\mathrm{CRI}_{16}$ & $20 \pm 0.14$ & $17 \pm 0.17$ & $0.61 \pm 0.06$ & $0.29 \pm 0.01$ \\
\hline $\mathrm{CRI}_{17}$ & $18 \pm 0.13$ & $17 \pm 0.18$ & $0.58 \pm 0.07$ & $0.30 \pm 0.03$ \\
\hline $\mathrm{CRI}_{18}$ & $19 \pm 0.21$ & $16 \pm 0.14$ & $0.59 \pm 0.09$ & $0.27 \pm 0.03$ \\
\hline $\mathrm{CRI}_{19}$ & $16 \pm 0.20$ & $15 \pm 0.09$ & $0.58 \pm 0.08$ & $0.25 \pm 0.04$ \\
\hline${ }^{*} \mathrm{CRI}_{20}$ & $23 \pm 0.12$ & $20 \pm 0.10$ & $0.67 \pm 0.08$ & $0.39 \pm 0.03$ \\
\hline $\mathrm{CRI}_{21}$ & $19 \pm 0.13$ & $18 \pm 0.11$ & $0.59 \pm 0.05$ & $0.31 \pm 0.04$ \\
\hline $\mathrm{CRI}_{22}$ & $19 \pm 0.22$ & $17 \pm 0.09$ & $0.60 \pm 0.06$ & $0.31 \pm 0.02$ \\
\hline $\mathrm{CRI}_{23}$ & $18 \pm 0.19$ & $17 \pm 0.08$ & $0.58 \pm 0.07$ & $0.28 \pm 0.03$ \\
\hline $\mathrm{CRI}_{24}$ & $20 \pm 0.12$ & $19 \pm 0.11$ & $0.61 \pm 0.10$ & $0.31 \pm 0.03$ \\
\hline $\mathrm{CRI}_{25}$ & $17 \pm 0.17$ & $15 \pm 0.14$ & $0.55 \pm 0.02$ & $0.24 \pm 0.02$ \\
\hline $\mathrm{CRI}_{26}$ & $18 \pm 0.18$ & $16 \pm 0.20$ & $0.54 \pm 0.08$ & $0.26 \pm 0.02$ \\
\hline $\mathrm{CRI}_{27}$ & $19 \pm 0.18$ & $17 \pm 0.14$ & $0.51 \pm 0.07$ & $0.27 \pm 0.02$ \\
\hline $\mathrm{CRI}_{28}$ & $20 \pm 0.19$ & $18 \pm 0.19$ & $0.60 \pm 0.09$ & $0.31 \pm 0.02$ \\
\hline $\mathrm{CRI}_{29}$ & $19 \pm 0.22$ & $17 \pm 0.18$ & $0.59 \pm 0.08$ & $0.28 \pm 0.01$ \\
\hline $\mathrm{CRI}_{30}$ & $20 \pm 0.18$ & $18 \pm 0.21$ & $0.58 \pm 0.07$ & $0.29 \pm 0.02$ \\
\hline $\mathrm{CRI}_{31}$ & $21 \pm 0.19$ & $17 \pm 0.22$ & $0.59 \pm 0.05$ & $0.30 \pm 0.03$ \\
\hline $\mathrm{CRI}_{32}$ & $19 \pm 0.19$ & $17 \pm 0.21$ & $0.57 \pm 0.04$ & $0.29 \pm 0.02$ \\
\hline $\mathrm{CRI}_{33}$ & $18 \pm 0.17$ & $17 \pm 0.20$ & $0.59 \pm 0.06$ & $0.28 \pm 0.02$ \\
\hline${ }^{*} \mathrm{CRI}_{34}$ & $22 \pm 0.13$ & $20 \pm 0.07$ & $0.69 \pm 0.04$ & $0.35 \pm 0.05$ \\
\hline${ }^{*} \mathrm{CRI}_{35}$ & $21 \pm 0.10$ & $19 \pm 0.20$ & $0.60 \pm 0.05$ & $0.37 \pm 0.03$ \\
\hline $\mathrm{CRI}_{36}$ & $19 \pm 0.18$ & $18 \pm 0.21$ & $0.58 \pm 0.03$ & $0.30 \pm 0.03$ \\
\hline $\mathrm{CRI}_{37}$ & $19 \pm 0.19$ & $15 \pm 0.22$ & $0.58 \pm 0.05$ & $0.31 \pm 0.02$ \\
\hline $\mathrm{CRI}_{38}$ & $18 \pm 0.20$ & $16 \pm 0.18$ & $0.54 \pm 0.06$ & $0.29 \pm 0.05$ \\
\hline $\mathrm{CRI}_{39}$ & $18 \pm 0.19$ & $17 \pm 0.17$ & $0.55 \pm 0.04$ & $0.28 \pm 0.05$ \\
\hline $\mathrm{CRI}_{40}$ & $19 \pm 0.18$ & $17 \pm 0.20$ & $0.59 \pm 0.07$ & $0.29 \pm 0.04$ \\
\hline
\end{tabular}

*Selected for further study on cumulative basis according to General Linear Model

\section{Characterization of strains}

The results of characterization about various traits (Table 2) showed that these strains have higher colonization of roots with $\mathrm{CRI}_{35}$ having the maximum root colonization ability followed by $\mathrm{CRI}_{14}, \mathrm{CRI}_{20}$ and $\mathrm{CRI}_{34}$. Strains $\mathrm{CRI}_{18}$ did not show phosphate solubilization ability while $\mathrm{CRI}_{20}$ had maximum ability compared to others. The results regarding production of IAA in the presence of L-tryptophan showed that $\mathrm{CRI}_{35}$ produced maximum indole acetic acid followed by $\mathrm{CRI}_{20}$. It has been observed that strains $\mathrm{CRI}_{34}$ and $\mathrm{CRI}_{35}$ were found positive regarding siderophores production. Strains $\mathrm{CRI}_{20}$ and $\mathrm{CRI}_{35}$ showed the production of exopolysaccharides when examined qualitatively. 


\section{Effect of inoculation on growth parameters of imidacloprid treated chickpea plants}

Data (Table 3) showed that non-significant change was recorded in plant height of imidacloprid treated and untreated
3). The strain $\mathrm{CRI}_{35}$ caused significant increase in root dry weight in the presence and absence of imidacloprid when compared with respective un-inoculated control treatment. The increases in root length over the respective uninoculated control in case of treated and untreated seeds

Table 2. Characterization of rhizobium strains (Average of four replications)

\begin{tabular}{llllll}
\hline Isolate & $\begin{array}{l}\text { Root colonization } \\
\text { (CFU per gram of } \\
\text { root) }\end{array}$ & $\begin{array}{l}\text { Phosphate } \\
\text { solubilization } \\
\text { Halo size }(\mathbf{c m})\end{array}$ & $\begin{array}{l}\text { Indole acetic acid } \\
(\boldsymbol{\mu g} / \mathbf{m L})\end{array}$ & $\begin{array}{l}\text { Siderophores } \\
\text { production }\end{array}$ & $\begin{array}{l}\text { Exopolysaccharides } \\
\text { production }\end{array}$ \\
\hline CRI $_{14}$ & $2.1 \times 10^{9}$ & - & $25.3 \pm 2.1$ & - & - \\
CRI $_{20}$ & $4.0 \times 10^{8}$ & $15.0 \pm 1.7$ & $32.8 \pm 2.8$ & - & + \\
CRI $_{34}$ & $3.5 \times 10^{7}$ & $10.5 \pm 1.2$ & $29.5 \pm 2.5$ & + & - \\
CRI $_{35}$ & $2.8 \times 10^{9}$ & $17.0 \pm 1.4$ & $39.0 \pm 3.4$ & ++ & + \\
\hline
\end{tabular}

Table 3. Effect of inoculation on plant height, plant biomass, root length and root dry weight of root of chickpea

\begin{tabular}{|c|c|c|c|c|c|c|c|c|}
\hline & \multicolumn{2}{|c|}{ Plant height (cm) } & \multicolumn{2}{|c|}{ Plant biomass (g) } & \multicolumn{2}{|c|}{ Root length (cm) } & \multicolumn{2}{|c|}{ Root dry weight (gm) } \\
\hline & $\mathbf{T}_{\mathbf{0}}$ & $\mathbf{T}_{1}$ & $\mathbf{T}_{\mathbf{0}}$ & $\mathbf{T}_{1}$ & $\mathbf{T}_{\mathbf{0}}$ & $\mathbf{T}_{1}$ & $\mathbf{T}_{\mathbf{0}}$ & $\mathbf{T}_{1}$ \\
\hline Control & $40.2 \mathrm{~d}$ & $35.9 \mathrm{e}$ & $41.4 \mathrm{e}$ & $35.4 \mathrm{f}$ & $21.5 \mathrm{bc}$ & $15.7 \mathrm{~d}$ & $1.86 \mathrm{~cd}$ & $1.67 \mathrm{~d}$ \\
\hline $\mathrm{CRI}_{14}$ & $41.5 \mathrm{~d}$ & $37.2 \mathrm{e}$ & $50.2 \mathrm{c}$ & $44.2 \mathrm{~d}$ & $28.2 \mathrm{a}$ & $22.7 \mathrm{~b}$ & $2.16 \mathrm{ab}$ & $2.01 \mathrm{bc}$ \\
\hline $\mathbf{C R I}_{20}$ & $48.2 \mathrm{ab}$ & $48.4 \mathrm{ab}$ & $53.3 \mathrm{~b}$ & $50.3 \mathrm{c}$ & $29.2 \mathrm{a}$ & $23.5 \mathrm{~b}$ & $2.06 \mathrm{bc}$ & $1.90 \mathrm{c}$ \\
\hline $\mathrm{CRI}_{34}$ & $44.3 \mathrm{c}$ & $36.1 \mathrm{e}$ & $48.9 \mathrm{~cd}$ & $42.7 \mathrm{de}$ & $26.7 \mathrm{ab}$ & $20.9 \mathrm{c}$ & $2.13 \mathrm{~b}$ & $2.10 \mathrm{~b}$ \\
\hline $\mathrm{CRI}_{35}$ & $50.6 \mathrm{a}$ & $48.9 \mathrm{ab}$ & $56.6 \mathrm{a}$ & $56.1 \mathrm{a}$ & $28.2 \mathrm{a}$ & $28.7 \mathrm{a}$ & $2.23 \mathrm{a}$ & $2.22 \mathrm{a}$ \\
\hline
\end{tabular}

$\mathbf{T}_{0^{-}}$- Untreated seed; $\mathbf{T}_{1^{-}}$Imidacloprid treated seed

chickpea. However, the inoculation with pesticide-tolerant M. ciceri significantly enhanced the plant height of chickpea when compared with un-inoculated control. Both strains were equally effective for improving the plant height of imidacloprid treated and un-treated chickpea plants and statistically similar results were obtained regarding plant height due to inoculation with $\mathrm{CRI}_{20}$ and $\mathrm{CRI}_{35}$.

A significant increase in fresh biomass of chickpea was observed due to inoculation in treated and untreated plants compared to un-inoculated ones (Table 3). Non-significant difference was observed in case of treated and untreated plants. Maximum improvement in fresh biomass $(38.60 \%)$ was observed due to inoculation with $\mathrm{CRI}_{35}$ in untreated plants followed by inoculation with same strain in imidacloprid treated plants i.e. $36.66 \%$.

A significant decrease in root length was observed in case of imidacloprid treated control plant compared to untreated ones (Table 3). Similarly, decrease in root length was also observed in case of inoculated treated plant when compared with untreated plants, however, this decrease was non-significant. Inoculation caused significant increase in the root length of chickpea as compared to respective uninoculated control in the presence and absence of imidacloprid treatment.

Root dry weight was significantly improved due to inoculation with $\mathrm{CRI}_{35}$ in insecticide treated plants (Table were 44.76 and $54.87 \%$, respectively.

Results (Table 4) showed that seed treatment with imidacloprid significantly decreased the number of pods plant $^{-1}$ except in case of treatment where seeds were inoculated with $\mathrm{CRI}_{35}$. Inoculation with pesticide tolerant $M$. ciceri strains significantly improved the pods formation compared to un-inoculated control. Maximum improvement in number of pods plant ${ }^{-1}$ was observed due to inoculation with $\mathrm{CRI}_{35}$ in imidacloprid treated plants.

\section{Effect of inoculation on nodulation and yield of imidacloprid treated chickpea plants}

Seed treatment of chickpea with imidacloprid adversely affected the nodulation process. Seed treatment significantly decreased the number of nodules when compared with untreated plants (Table 4). However, inoculation with pesticide tolerant $M$. ciceri strains reduced the negative effect of imidacloprid treatment on nodulation and significantly improved the number of nodules. The results of strain $\mathrm{CRI}_{35}$ were more pronounced in imidacloprid treated plants where it gave significantly better performance than $\mathrm{CRI}_{20}$. The increase in number of nodules due to $\mathrm{CRI}_{35}$ was $93 \%$ more compared to respective un-inoculated control. The results of inoculation were also convincing in untreated plants and significant increase in number of nodules was observed in inoculated plants compared to un-inoculated ones. The effect of inoculation was also significant regarding nodule dry 
weight (Table 4). Both strains $\mathrm{CRI}_{20}$ and $\mathrm{CRI}_{35}$ showed equally good results which were significantly better than respective un-inoculated control.

Similar to number of pods, the grain yield pot ${ }^{-1}$ was also decreased due to imidacloprid treatment in the absence of inoculation (Table 4). Both the strains, $\mathrm{CRI}_{20}$ and $\mathrm{CRI}_{35}$, significantly improved the grain yield pot $^{-1}$ in imidacloprid treated and untreated plants. The results were more prominent in case of inoculation with $\mathrm{CRI}_{35}$ where the improvements in grain yield pot ${ }^{-1}$ were 92 and $82 \%$ in imidacloprid treated and untreated plants, respectively.

Both strains showed non-significant results regarding 100-grain weight of imidacloprid treated and untreated chickpea plants however, it was significantly higher when compared with un-inoculated control (Table 4). Inoculation significantly improved the 100-grain weight of chickpea as however, it was statistically similar with other treatments. In case of shoot, the highest phosphorus content was obtained with $\mathrm{CRI}_{35}$ that was $24 \%$ than un-inoculated control.

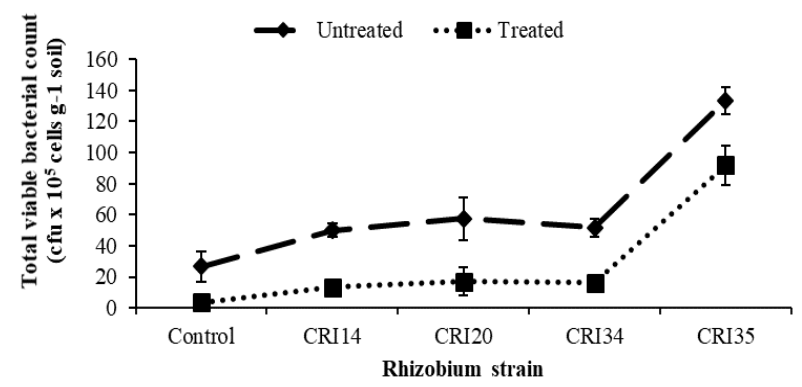

Figure 2: Impact of imidacloprid on bacterial population in chickpea rhizosphere

Table 4: Effect of rhizobial inoculation on number of nodules, number of pods, grain yield and 100 grain weight of chickpea

\begin{tabular}{lcccccccccc}
\hline & $\begin{array}{c}\text { No. of nodules per } \\
\text { plant }\end{array}$ & \multicolumn{2}{c}{$\begin{array}{c}\text { Nodules dry weight } \\
(\mathbf{g m})\end{array}$} & \multicolumn{2}{c}{$\begin{array}{c}\text { No. of pods per } \\
\text { plant }\end{array}$} & \multicolumn{2}{c}{$\begin{array}{c}\text { Grain yield per pot } \\
(\mathbf{g m})\end{array}$} & \multicolumn{2}{c}{$\begin{array}{c}\mathbf{1 0 0} \text { grain weight } \\
\text { (gm) }\end{array}$} \\
\hline & $\mathbf{T}_{\mathbf{0}}$ & $\mathbf{T}_{\mathbf{1}}$ & $\mathbf{T}_{\mathbf{0}}$ & $\mathbf{T}_{\mathbf{1}}$ & $\mathbf{T}_{\mathbf{0}}$ & $\mathbf{T}_{\mathbf{1}}$ & $\mathbf{T}_{\mathbf{0}}$ & $\mathbf{T}_{\mathbf{1}}$ & $\mathbf{T}_{\mathbf{0}}$ & $\mathbf{T}_{\mathbf{1}}$ \\
$\mathbf{C}$ & $32.6 \mathrm{~d}$ & $28.0 \mathrm{e}$ & $0.23 \mathrm{e}$ & $0.19 \mathrm{f}$ & $45 \mathrm{c}$ & $39 \mathrm{e}$ & $6.9 \mathrm{~d}$ & $5.5 \mathrm{e}$ & $31.2 \mathrm{~b}$ & $30.1 \mathrm{bc}$ \\
$\mathbf{C R I}_{\mathbf{1 4}}$ & $48.0 \mathrm{~b}$ & $44.9 \mathrm{c}$ & $0.29 \mathrm{~cd}$ & $0.27 \mathrm{de}$ & $50 \mathrm{bc}$ & $41 \mathrm{~d}$ & $9.8 \mathrm{bc}$ & $8.4 \mathrm{c}$ & $35.4 \mathrm{ab}$ & $34.9 \mathrm{ab}$ \\
$\mathbf{C R I}_{\mathbf{2 0}}$ & $52.3 \mathrm{a}$ & $49.0 \mathrm{~b}$ & $0.33 \mathrm{~b}$ & $0.31 \mathrm{bc}$ & $55 \mathrm{~b}$ & $52 \mathrm{~b}$ & $10.0 \mathrm{~b}$ & $9.6 \mathrm{bc}$ & $36.0 \mathrm{a}$ & $34.5 \mathrm{ab}$ \\
$\mathbf{C R I}_{\mathbf{3 4}}$ & $51.4 \mathrm{ab}$ & $45.0 \mathrm{c}$ & $0.33 \mathrm{~b}$ & $0.30 \mathrm{c}$ & $53 \mathrm{~b}$ & $45 \mathrm{c}$ & $10.2 \mathrm{ab}$ & $9.4 \mathrm{bc}$ & $36.1 \mathrm{a}$ & $36.4 \mathrm{a}$ \\
$\mathbf{C R I}_{\mathbf{3 5}}$ & $53.6 \mathrm{a}$ & $49.3 \mathrm{~b}$ & $0.37 \mathrm{a}$ & $0.36 \mathrm{a}$ & $62 \mathrm{a}$ & $59 \mathrm{a}$ & $12.0 \mathrm{a}$ & $10.4 \mathrm{a}$ & $37.9 \mathrm{a}$ & $36.2 \mathrm{a}$ \\
\hline
\end{tabular}

$\mathrm{T}_{0^{-}}$Untreated seed; $\mathrm{T}_{1}$ - Imidacloprid treated seed

Table 5: Effect of rhizobial inoculation on nitrogen and phosphorus contents of imdacloprid treated chickpea

\begin{tabular}{lcccccccc}
\hline Strains & \multicolumn{3}{c}{ Nitrogen (N) content (mg/g) } & \multicolumn{3}{c}{ Phosphorus (P) content (mg/g) } \\
& \multicolumn{3}{c}{ Root } & \multicolumn{2}{c}{ Shoot } & \multicolumn{2}{c}{ Root } & \multicolumn{2}{c}{ Shoot } \\
\cline { 2 - 9 } & $\mathbf{T}_{\mathbf{0}}$ & $\mathbf{T}_{\mathbf{1}}$ & $\mathbf{T}_{\mathbf{0}}$ & $\mathbf{T}_{\mathbf{1}}$ & $\mathbf{T}_{\mathbf{0}}$ & $\mathbf{T}_{\mathbf{1}}$ & $\mathbf{T}_{\mathbf{0}}$ & $\mathbf{T}_{\mathbf{1}}$ \\
Control & $39.7 \mathrm{c}$ & $33.9 \mathrm{~d}$ & $55.6 \mathrm{bc}$ & $45.5 \mathrm{c}$ & $0.28 \mathrm{bc}$ & $0.25 \mathrm{c}$ & $0.31 \mathrm{c}$ & $0.28 \mathrm{e}$ \\
CRI $_{14}$ & $40.2 \mathrm{bc}$ & $35.5 \mathrm{~cd}$ & $58.9 \mathrm{ab}$ & $54.7 \mathrm{bc}$ & $0.30 \mathrm{~b}$ & $0.25 \mathrm{c}$ & $0.39 \mathrm{a}$ & $0.33 \mathrm{bc}$ \\
CRI $_{20}$ & $42.5 \mathrm{~b}$ & $40.1 \mathrm{bc}$ & $60.2 \mathrm{a}$ & $58.2 \mathrm{ab}$ & $0.33 \mathrm{a}$ & $0.29 \mathrm{~b}$ & $0.36 \mathrm{ab}$ & $0.31 \mathrm{c}$ \\
CRI $_{\mathbf{3 4}}$ & $41.5 \mathrm{~b}$ & $40.5 \mathrm{~b}$ & $60.7 \mathrm{a}$ & $56.7 \mathrm{~b}$ & $0.32 \mathrm{ab}$ & $0.24 \mathrm{c}$ & $0.41 \mathrm{a}$ & $0.35 \mathrm{~b}$ \\
CRI $_{35}$ & $45.0 \mathrm{a}$ & $44.5 \mathrm{a}$ & $61.9 \mathrm{a}$ & $58.8 \mathrm{ab}$ & $0.34 \mathrm{a}$ & $0.27 \mathrm{bc}$ & $0.40 \mathrm{a}$ & $0.37 \mathrm{ab}$ \\
\hline
\end{tabular}

$\mathbf{T}_{0^{-}}$Untreated seed; $\mathbf{T}_{\mathbf{1}^{-}}$Imidacloprid treated seed

compared to respective un-inoculated control in the presence and absence of imidacloprid treatment.

Inoculation enhanced the nitrogen and phosphorus contents in both root and shoot of chickpea plant in imidacloprid treated and untreated plants (Table 5). Up to 31 and $11 \%$ increases in nitrogen content of root over control were recorded with $\mathrm{CRI}_{35}$ in imidacloprid treated and untreated plants respectively. In case of shoot, the increases in nitrogen content with same strain were 22 and $11 \%$ in treated and untreated treatment. Phosphorus contents of chickpea root was $16 \%$ more $\mathrm{CRI}_{20}$ than un-inoculated in treated plants

\section{Bacterial population density in chickpea rhizosphere}

Treatment with imidacloprid also adversely affected the bacterial population in the chickpea rhizosphere where a significant decrease in total viable bacterial count was observed when compared with untreated control (Figure 2). However, application of pesticide-tolerant rhizobial strains maintained the growth of viable bacteria in the rhizosphere and significant improvement in the viable bacterial cell was observed in pesticide-treated plant. The strain $\mathrm{CRI}_{35}$ showed more prominent results than $\mathrm{CRI}_{20}$ and the results were 
significantly better when compared to $\mathrm{CRI}_{20}$ and respective un-inoculated control in imidacloprid treated and untreated plants.

\section{Discussion}

In the present study, among four rhizobial strains those were assessed for their potential to tolerate pesticide, two strains $\mathrm{CRI}_{20}$ and $\mathrm{CRI}_{35}$ showed tolerance against higher levels of imidacloprid while the other strains $\mathrm{CRI}_{14}$ and $\mathrm{CRI}_{34}$ were sensitive to pesticide even when tested at lower levels. The mechanism of pesticide tolerance in microbes is a complex process which depends upon the physiology and genetics of the microorganisms. The microbes those having potential to degrade the pesticide (organic compound to meet their carbon requirement) showed resistance against them (Ortiz-Hernandez and Sanchez-Salinas, 2010). The insecticide tolerance of rhizobial strains might be due to their ability to use imidacloprid as a carbon source for their metabolism because there was no other carbon source in that medium supplied to bacterial strains for their growth. The variation in tolerance to imidacloprid by rhizobium strains might be due to various strategies adopted by the bacteria such as biodegradation and enzyme hydrolysis to overcome the toxic effect of chemicals (Herman et al., 2005; Yang and Lee, 2008). The tolerance against a pesticide may be temporary one that is due to induction of new metabolic pathway to bypass a biochemical reaction that could inhibit the growth of microbe (Bellinaso et al., 2003). Permanent resistance, on the other hand, depends on genetic modifications, inherited by the subsequent generation of microbes (Herman et al., 2005). The better tolerance of $\mathrm{CRI}_{20}$ and $\mathrm{CRI}_{35}$ compared to other strains might be due to their ability to produce exopolysaccharides (EPS). As the EPS provides protection to bacteria against environmental stress (Tank and Saraf, 2003), it is possible that bacteria secreted more EPS in the presence of insecticide to shield themselves against toxic effect of insecticide. It is also evident from the work of Ahemad and Khan (2012) where Mesorhizobium sp. when exposed to high concentration of pesticide produced more EPS compared to its low concentration.

Pesticides not only damage structural proteins essential for the microbial growth but are also responsible for genotoxicity (Pham et al., 2004). The application of pesticides at high concentration decreases functioning and survival of organisms (Kumar et al., 2010). In our studies, imidacloprid treatment to chickpea seeds at normal rate showed nonsignificant effect on growth parameters while a significant decrease in nodulation, growth and yield parameters of chickpea was observed. This might be due to toxic effects of pesticide on microbial activity and nitrogen fixing ability which ultimately leads to decrease in yield of chickpea plants. A decrease in plant growth and yield due to pesticide application has also been observed in earlier studies owing to negative effects of pesticides on indigenous microbial population (Guene et al., 2003) and enzyme inhibition (Zablotowicz and Reddy, 2004) that lead to decrease in nitrogen fixation (Fox et al., 2007). It has been observed that use of chemicals like fungicides, to protect plant from diseases, are toxic to rhizobial growth and viability, even if rhizobia remain viable, its efficiency to fix nitrogen can be reduced (Zahran, 1999; Guene et al., 2003, Ahemad and Khan, 2013).

Soil fertility is directly related to microbial processes which are responsible to supply nutrients for plant growth (Smith, 1991). Nitrogen is the most important and limiting nutrient in plant nutrition which is directly related to population and symbiosis of rhizobia in the soil. So, nodulation and soil microbial population are the important parameters to describe the effect of pesticides on soil fertility. In our studies, imidacloprid has significantly decreased the nodulation and soil bacterial population. The inhibitory effect on these parameters may be attributed to the toxicity of imidacloprid for indigenous rhizobial strains and rhizosphere microflora. However, this inhibitory effect was diluted due to inoculation with pesticide tolerant Mesorhizobium sp. Similar results have also been reported by other workers (Yang and Lee, 2008; Zaidi et al., 2009, Ahemad and Khan, 2009, 2012a).

Interestingly, inoculation with Mesorhizobium strains improved the plant growth and yield in imidacloprid treated and untreated plants, however, with variable degree of efficacy. The more significant results of these strains in imidacloprid treated plants may be due to detoxification of imidacloprid (Yang and Lee, 2008) and also due to other direct or indirect plant growth promoting characters (Zaidi et al., 2009). Hence, this trait confers the selective advantage to compete with other soil microflora (Ayansina, 2009). The detoxification potential of these strains is also supported by their prolific growth on minimal media having imidacloprid as carbon source. In our study, the significant improvement in growth and yield parameters in the presence of insecticide was might be due to the tolerant ability of rhizobium strain against imidacloprid. The presence of these insecticide tolerant strains reduced the toxic effect of insecticide on chickpea. Consequently, M. ciceri improved the growth, yield and nodulation in chickpea plants by protecting the chickpea from imidacloprid toxicity. It is also evident from the work of Ahemad and Khan (2009). The improvement of plant growth yield and nodulation may also be attributed to other growth promoting traits such as IAA production, phosphate solubilization, exopolysaccharides and siderophores synthesis which play important role in 
plant growth promotion. For example, exopolysaccharides production helps in nitrogen fixation by preventing high oxygen tension (Tank and Saraf, 2003). The better growth of $\mathrm{CRI}_{20}$ and $\mathrm{CRI}_{35}$ might be due to the reason that exopolysaccharides provide it protection against the stressed environments and ultimately enable it to enhance plant growth compared to other strains. Earlier work of Ahemad and Khan (2012b) also showed that exopolysaccharides producing Pseudomonas showed better growth in fungicide treated environment.

\section{Conclusion}

It is concluded that inoculation with pesticide tolerant rhizobial strains (M. ciceri) improved the chickpea growth and yield. Therefore, such strains can be used in conditions where seed treatment is done with insecticide. However, further evaluation of these strains under farmer's field conditions is needed so that suitable biofertilizer can be prepared for improving chickpea growth on sustainable basis in pesticide-treated soils.

\section{Acknowledgment}

The authors are thankful to the Soil Microbiology \& Biochemistry lab, Institute of Soil \& Environmental Sciences, University of Agriculture, Faisalabad for providing facilities to complete this work.

\section{References}

Ahemad, M. and M.S. Khan. 2009. Effect of insecticidetolerant and plant growth-promoting Mesorhizobium on the performance of chickpea grown in insecticide stressed alluvial soils. Journal of Crop Science and Biotechnology 12: 217-226.

Ahemad, M. and M.S. Khan. 2010. Influence of selective herbicides on plant growth promoting traits of phosphate solubilizing Enterobacter esburiae strain PS2. Research Journal of Microbiology 5: 849-857.

Ahemad, M. and M.S. Khan. 2011. Ecotoxicological assessment of pesticides towards the plant growth promoting activities of Lentil (Lens esculentus)-specific Rhizobium sp. strain MRL3. Ecotoxicology 20: 661669.

Ahemad, M. and M.S. Khan. 2012a. Effects of pesticides on plant growth promoting traits of Mesorhizobium strain MRC4. Journal of the Saudi Society of Agricultural Sciences 11: 63-71.

Ahemad, M. and M.S. Khan. 2012b. Effect of fungicides on plant growth promoting activities of phosphate solubilizing Pseudomonas putida isolated from mustard (Brassica compestris) rhizosphere. Chemosphere 86: 945-950
Ahemad, M. and M.S. Khan. 2013. Pesticides as antagonists of rhizobia and the legume-rhizobium symbiosis: A paradigmatic and mechanistic outlook. Biochemistry \& Molecular Biology 1(4): 63-75.

Alexander, D.B. and D.A. Zuberer. 1991. Use of chrome azurol $\mathrm{S}$ reagents to evaluate siderophore production by rhizosphere bacteria. Biology and Fertility of Soils 12: $39-45$.

Ali, A., Z. Ali, J. Iqbal, M.A. Nadeem, N. Akhtar, H.M. Akram and A. Sattar. 2010. Impact of nitrogen and phosphorus on seed yield of chickpea. Journal of Agricultural Research 48:335-343

Anderson, A., J.A. Baldock, S.L. Rogers, W. Bellotti and G. Gill. 2004. Influence of chlorsulfuron on rhizobial growth, nodule formation, and nitrogen fixation with chickpea, Australian Journal of Agricultural Research 55: 1059-1070.

Ayansina, A.D.V. 2009. Pesticide use in agriculture and microorganisms. P. 261-284. In: Microbes in Sustainable Agriculture. M.S. Khan, (Eds). Nova Science Publishers Inc, New York, USA.

Ayers, R.S. and D.W. Westcot. 1985. Water quality for agriculture. FAO Irrigation and Drainage Papers 29(Rev 1), FAO, Rome, Italy.

Bellinaso, M.L., C.W. Greer, M.C. Peralba, J.A. Henriques and C.C. Gaylarde. 2003. Biodegradation of the herbicide trifluralin by bacteria isolated from soil. FEMS Microbiology Ecology 43:191-194.

Binyamin, R., S.M. Nadeem, S. Akhtar, M.Y. Khan and R. Anjum. 2019. Beneficial and pathogenic plant-microbe interactions: A review. Soil \& Environment 38: $127-$ 150.

Brick, J.M., R.M. Bostock and S.E. Silversone. 1991. Rapid in situ assay for indole acetic acid production by bacteria immobilized on nitrocellulose membrane. Applied Environmental Microbiology 57:535-538.

Datta, A., B.M. Sindel, P. Kristiansen, R.S. Jessop and W.L. Felton. 2009. Effect of isoxaflutole on the growth, nodulation and nitrogen fixation of chickpea (Cicer arietinum L.). Crop Protection 28: 923-927.

Dubey, R.C., D.K. Maheshwari, H. Kumar and K. Choure. 2010. Assessment of diversity and plant growth promoting attributes of rhizobia isolated from Cajanus cajan L. African Journal of Biotechnology 9:86198629.

Eliason, R., J.J. Schoenau, A.M. Szmigielski and W.M. Laverty. 2004. Phytotoxicity and persistence of flucarbazone-sodium in soil. Weed Science 52: 857862.

Fox, J.E., J. Gulledge, E. Engelhaupt, M.E. Burow and J.A. McLachlan. 2g007. Pesticides reduce symbiotic efficiency of nitrogen-fixing rhizobia and host plants. 
Proceedings of the National Academy of Sciences 104:10282-10287.

Gaind, S., M.S. Rathi, B.D. Kaushik, L. Nain and O.P. Verma. 2007. Survival of bio-inoculants on fungicides treated seeds of wheat, pea and chickpea and subsequent effect on chickpea yield. Journal of Environmental Science and Health 42:663-668.

Guene, N.F.D., A. Diouf and M. Gueye. 2003. Nodulation and nitrogen fixation of field grown common bean (Phaseolus vulgaris) as influenced by fungicide seed treatment. African Journal of Biotechnology 2:198-201.

Herman, P.L., M. Behrens, S. Chakraborty, B.M. Chrastil, J. Barycki, and D.P. Weeks. 2005. A three-component dicamba O-demethylase from Pseudomonas maltophilia, strain DI-6 gene isolation, characterization, and heterologous expression. Journal of Biological Chemistry 280: 24759-24767.

Herman, P.L., M. Behrens, S. Chakraborty, B.M. Crastil, J. Barycki and D.P. Weeks. 2005. A three component dicamba O-demethylase from Pseudomonas maltophilia strain DI-6, gene isolation, characterization and heterologous expression. Journal Biological Chemistry 280:24759-24767.

Koopman, D.J., P.G. Tow, T.G. Reeves and A.H. Gibson. 1995. Soil acidification, chlorsulfuron application and Rhizobium melilotias factors in lucerne yield decline. Soil Biology and Biochemistry 27: 673-677.

Kumar, N.J.I., A. Bora\& and M.K. Amb. 2010. Chronic toxicity of the triazole fungicide tebuconazole on a heterocystous, nitrogen-fixing rice paddy field cyanobacterium, Westiellopsis prolifica Janet. Journal of Microbiology and Biotechnology 20:1134-1139.

Li, J.F., S.Q. Zhang, P.H. Huo, S.L. Shi and Y.Y. Miao. 2013. Effect of phosphate solubilizing rhizobium and nitrogen fixing bacteria on growth of alfalfa seedlings under $\mathrm{P}$ and $\mathrm{N}$ deficient conditions. Pakistan Journal of Botany 45: 1557-1562.

Mead, R., R.N. Curnow and A.M. Hasted. 2003. Statistical methods in agriculture and experimental botany. 3rd Ed. Chapman and Hall/CRS Press, Boca Raton, Fla.

Mohammadi, K., A. Ghalavand and M. Aghaalikhani. 2010. Effect of organic matter and biofertilizers on chickpea quality and biological nitrogen fixation. World Academy of Science, Engineering and Technology 44:1154-1159.

Nicolaus, B., L.Lama, E. Esposito, M.C. Manca, R. Improta, M.R. Bellitti, A.W. Duckworth, W.D. Grant and A. Gambacorta. 1999. Haloarculaspp able to biosynthesize exo-endopolymers. Journal of Industrial Microbiology and Biotechnology 23: 489-496.

Ortiz-Hernandez, M.L. and E. Sanchez-Salinas. 2010. Biodegradation of the organophosphate pesticide tetrachlorvinphos by bacteria isolated from agricultural soils in Mexico. Revista Internacional de Contaminación Ambiental 26: 27-38.

Parween, T., S. Jan, S. Mahmooduzzafar, T. Fatma and Z.H. Siddiqui. 2016. Selective effect of pesticides on plantA Review. Critical Reviews in Food Science and Nutrition 56: 160-179.

Pham, C.H., J. Min and M.B. Gu. 2004. Pesticide induced toxicity and stress response in bacterial cells. Bulletin of Environmental Contamination and Toxicology 72:380386.

Pikovskaya, R.I.1948. Mobilization of phosphorus in soil in connection with vital activity of some microbial species. Microbiology 17:362-370.

Russell, A.D., W.B. Hugo and G.A.J. Ayliffo. 1982. Principles and practices of disinfection, preservation and sterilization. Black Wall Scientific, London.

Ryan, J., G. Estefan and A. Rashid. 2001.Soil and plant analysis: laboratory manual. International Centre for Agricultural Research in Dry Areas (ICARDA), Aleppo, Syria.

Sahasrabudhe, M.M. 2011. Screening of rhizobia for indole acetic acid production. Annals of Biological Research 2:460-468.

Simons, M., A.J.van der Bij,I. Brand, L.A. de Weger,C.A. Wijffelman and B.J.J. Lugtenberg. 1996. Gnotobiotic system for studying rhizosphere colonization by plant growth-promoting Pseudomonas bacteria. Molecular Plant-Microbe Interactions 9: 600-607.

Smith, S.R. 1991. Effects of sewage sludge application on soil microbial processes and soil fertility. In: Advances in soil science. Stewart, B.A. (Ed.) Springer-Verlag, NY. 191-212 p.

Somasegaran, P. and H.J. Hoben. 1994. Handbook for Rhizobia: methods in legume rhizobium technology. Springer, New York, USA.

Steel, R.G.D., J.H. Torrie and A.D. Dickey. 2007. Principles and procedures of statistics: a biometrical approach, 3rd Edn. McGraw Hill Book Co, New York.

Tank, N. and M. Saraf. 2003. Phosphate solubilization, exopolysaccharide production and indole acetic acid secretion by rhizobacteria isolated from Trigonella foenum-graecum, Indian Journal of Microbiology 43: 37-40.

Vincent, J.M. 1970. A manual for the Practical Study of Root Nodule bacteria. IBP Handbook 15: Blackwell Scientific Publications, Oxford and Edinburgh, England.

Wani, P.A., A. Zaidi, A.A. Khan and M.S. Khan. 2005. Effect of phorate on phosphate solubilization and indole acetic acid releasing potentials of rhizospheric 
microorganisms. Annals of Plant Protection Sciences 13:139-144.

Yang, C. and C. Lee. 2008. Enrichment, isolation, and characterization of 4-chlorophenol-degrading bacterium Rhizobium spp. 4-CP-20. Biodegradation 19:329-336.

Yang, C.F. and C.M. Lee. 2008. Enrichment, isolation, and characterization of 4-chlorophenol-degrading bacterium Rhizobium sp. 4-CP-20. Biodegradation 19: 329-336.

Zablotowicz, R.M. and K.N. Reddy. 2004. Impact of glyphosate on the Bradyrhizobium japonicum symbiosis with glyphosate-resistant transgenic soybean: a mini review. Journal of Environmental Quality $33: 825-831$.
Zahir, Z.A., M. Ahmad, T.H. Hilger, A. Dar, S.R. Malik, G. Abbas and F. Rasche. 2018. Field evaluation of multistrain biofertilizer for improving the productivity of different mungbean genotypes. Soil \& Environment 37: $45-52$

Zahran, H.H. 1999. Rhizobium-legume symbiosis and nitrogen fixation under sever conditions and in an Arid climate. Microbiology and Molecular Biology Reviews 63:968-989.

Zaidi, A., M.S. Khan, M. Ahemad and M. Oves. 2009. Plant growth promotion by phosphate solubilizing bacteria. Acta Microbiologica Et Immunologica Hungarica 56: 263-284. 